\title{
Debitagem laminar no Sul do Brasil: Habemus nucleos!
}

\author{
Antoine Lourdeau ${ }^{1}$, Mirian Carbonera ${ }^{2}$, Sirlei Hoeltz ${ }^{3}$, Marcos C. Pereira \\ Santos ${ }^{4}$, Lívia de Oliveira e Lucas ${ }^{5}$, Amélie Da Costa ${ }^{6}$, Sibeli Viana ${ }^{7}$
}

1. Muséum National d’Histoire Naturelle \& Universidade Federal de Sergipe. Musée de l’Homme, Département de Préhistoire, 17, place du Trocadéro, 75116 Paris, França. Email: antoine.lourdeau@mnhn.fr

2. Centro de Memória do Oeste de Santa Catarina (CEOM) \& Ciências Ambientais, Unochapecó, Rua John Kennedy, 279E, Chapecó - SC, Brasil. Email: mirianc@unochapeco.edu.br

3. Archaeo Pesquisas Arqueológicas, Av. Carandaí, 99, Parque Georgia, 78.085-485, Cuiabá - MT, Brasil. Email: sirlei@archaeo.com.br

4. Università Degli Studi di Ferrara \& Universidade do Extremo Sul Catarinense. Rodovia Governador Jorge Lacerda, km 4,5 - Bairro Sangão. 88805-350 - Caixa Postal 3167, Criciúma - SC, Brasil.

Email: marcoscesar@unesc.net

5. Universidade Federal de Sergipe \& Universidade Federal do Vale do São Francisco. UNIVASF, rua João

Ferreira dos Santos, s/nº, Campestre, 64770-000, São Raimundo Nonato - PI, Brasil.

Email: livia.lucas@univasf.edu.br

6. Université de Paris Ouest Nanterre La Défense. UMR 7041 ArScAn-ANTET. Maison de l’Archéologie et de

l’Ethnologie, 21 allée de l’Université, 92023 Nanterre, França. Email : amelie.da-costa@mae.u-paris10.fr

7. Pontifícia Universidade Católica de Goiás, Instituto Goiano de Pré-História e Antropologia, Praça

Universitária, n. 1440, Universitário, 74605-220, Goiania, GO - Brasil. Email: sibeli@pucgoias.edu.br

\section{Resumo:}

Durante a pré-história, a debitagem laminar é um modo de produção característico de determinados períodos e regiões. No Brasil, no momento, foi reconhecida em três sítios arqueológicos situados no alto vale do rio Uruguai (SC \& RS), em contextos sedimentares que datam do Holoceno inicial. Até hoje, somente os produtos dessa debitagem, as lâminas, tinham sido encontrados. A falta dos núcleos impedia um entendimento completo do processo de lascamento desses suportes. Graças à retomada das pesquisas na região desde 2013, foi possível coletar núcleos associados a essa produção laminar. Nesse artigo, descrevemos cinco desses núcleos. Suas análises demonstram que essa debitagem laminar corresponde a um único conceito. A estrutura volumétrica do núcleo que resulta desse conceito respeita as seguintes normas: 1) Os núcleos apresentam duas superfícies: um dorso plano e uma superfície de debitagem convexa mas relativamente achatada. Em uma extremidade, uma pequena superfície plana serviu de plano de percussão; 2) A debitagem começa por uma fase de inicialização durante a qual o plano de percussão é produzido por uma grande retirada. Em função das propriedades naturais do volume inicial, o dorso do núcleo é obtido, seja durante a seleção do bloco, seja por uma preparação por grandes retiradas transversais. Quando existem ainda, os negativos de retiradas da inicialização da superfície de debitagem indicam um método centrípeto; 3) As lâminas são sempre produzidas por um método unidirecional paralelo. A variabilidade desta debitagem é principalmente relacionada à técnica de lascamento: a percussão com pedra e a percussão com percutor orgânico foram utilizadas para a obtenção das lâminas. As informações tecnológicas fornecidas pelos núcleos aqui analisados são coerentes com essas observações oriundas da análise das lâminas. Com esses dois estudos consegue-se atingir um conhecimento completo desse sistema de

Published by the School of History, Classics and Archaeology, University of Edinburgh ISSN: 2055-0472. URL: http://journals.ed.ac.uk/lithicstudies/

This work is licensed under a Creative Commons Attribution 2.5 UK: Scotland License. 
produção do alto vale do rio Uruguai durante o Holoceno inicial, a única debitagem laminar documentada até hoje no Brasil.

Palavras-chave: debitagem laminar; pré-história do Brasil; rio Uruguai; Holoceno antigo; análise tecnológica; Foz do Chapecó

\section{Introdução}

A ocorrência da produção em série de lascas nitidamente alongadas ("lâminas"), ou seja, da debitagem laminar, é já bem conhecida no cone sul da América meridional, especificamente na Argentina (Barros et al. 2014; Hermo \& Magnin 2012; Hoguin 2013; Nami 1996) e no Uruguai (Suarez 2011) em contextos datados da primeira metade do Holoceno. No Brasil, as evidências são ainda muito raras e foram encontradas recentemente (Caldarelli 2010; Hoeltz \& Brüggeman 2011; 2014). Concentram-se principalmente em três sítios vizinhos, todos no alto vale do rio Uruguai, entre os estados de Santa Catarina (SC) e Rio Grande do Sul (RS) (Sul do Brasil) (Figura 1). Nesta região o rio Uruguai faz uma extensa curva, conhecida como Volta Grande, e o rio Chapecó, importante afluente do Uruguai, deságua no mesmo. Os três sítios, Alto Alegre 3 (ALP-AA-03), Linha Policial 1 (ACH-LP-01) e Linha Policial 3 (ACH-LP-03), foram alvo de pesquisas de salvamento relacionados ao licenciamento ambiental para construção da Usina Hidrelétrica Foz do Chapecó, entre 2006 e 2010 (Caldarelli 2010). Em todos, níveis arqueológicos datados do Holoceno inicial com lâminas foram encontrados. Nestes níveis, vários outros esquemas de produção líticos estão associados a essa debitagem laminar: façonnage de peças bifaciais de grande porte e de pontas de projétil, debitagem unipolar de lascas grandes, debitagem bipolar de lascas pequenas. As matérias-primas utilizadas são principalmente o arenito silicificado, a calcedônia e o quartzo hialino, provenientes de seixos do rio e de blocos extraídos nos arredores (Hoeltz \& Brüggemann 2011; 2014).

A presença dessa produção de lâminas logo chamou a atenção dos pesquisadores, mas o entendimento do esquema de produção destas peças ficava limitado, devido à ausência, nos três sítios, de núcleos provenientes das cadeias operatórias de debitagem laminar. Tal ausência pode ser o resultado de diferentes fatores. Em primeiro lugar, devido a suas propriedades de recorrência, a debitagem laminar caracteriza-se, muitas vezes, por uma importante produtividade, ou seja, pelo fato que um núcleo pode gerar uma importante quantidade de lâminas. Sendo assim, a escassez dos núcleos em relação a seus produtos não é surpreendente. Além disso, uma superfície reduzida dos sítios foi escavada (ao redor de 10\%) (Caldarelli 2010). É possível que as áreas de produção das lâminas, onde foram deixados os núcleos e outros resíduos de lascamento, não tenham sido atingidos pelas escavações.

Apesar da ausência dos núcleos, a partir de uma análise tecnológica detalhada das lâminas, baseada na interpretação dos esquemas diacríticos das mesmas, foi possível definir certas especificidades desse esquema de produção (Lourdeau et al. 2014; Hoeltz et al. 2015). Esse estudo demonstrou que tais suportes alongados foram produzidos a partir de diferentes técnicas de lascamento (percussão com pedra e percussão com percutor orgânico), mas seguindo sempre o mesmo método: uma inicialização centrípeta do núcleo nas partes onde era necessário modificar as propriedades naturais do bloco e, em seguida, uma produção unidirecional das lâminas. As modificações posteriores dos suportes oriundos desta debitagem, os retoques, permitem constatar que eles não eram destinados a uma única função. Eram suportes de instrumentos diversificados. A partir dos resultados das análises das lâminas, foi proposta uma estrutura hipotética dos núcleos dessa produção. Notou-se que os mesmos deviam apresentar um único plano de percussão, uma superfície de debitagem com convexidades laterais e distal marcadas para poder controlar o comprimento importante e a 
largura reduzida dos produtos, assim como a recorrência característica da debitagem laminar. Deviam apresentar também uma delimitação em dois planos distintos entre a superfície de debitagem e o resto do volume para permitir a retirada das lascas de inicialização segundo um método centrípeto, não se tratando então de uma debitagem volumétrica stricto sensu envolvendo todas as superfícies dos núcleos. Assim sendo, esses núcleos deviam ter um “dorso” oposto à superfície de debitagem, para o qual não se tinha informação tecnológica.

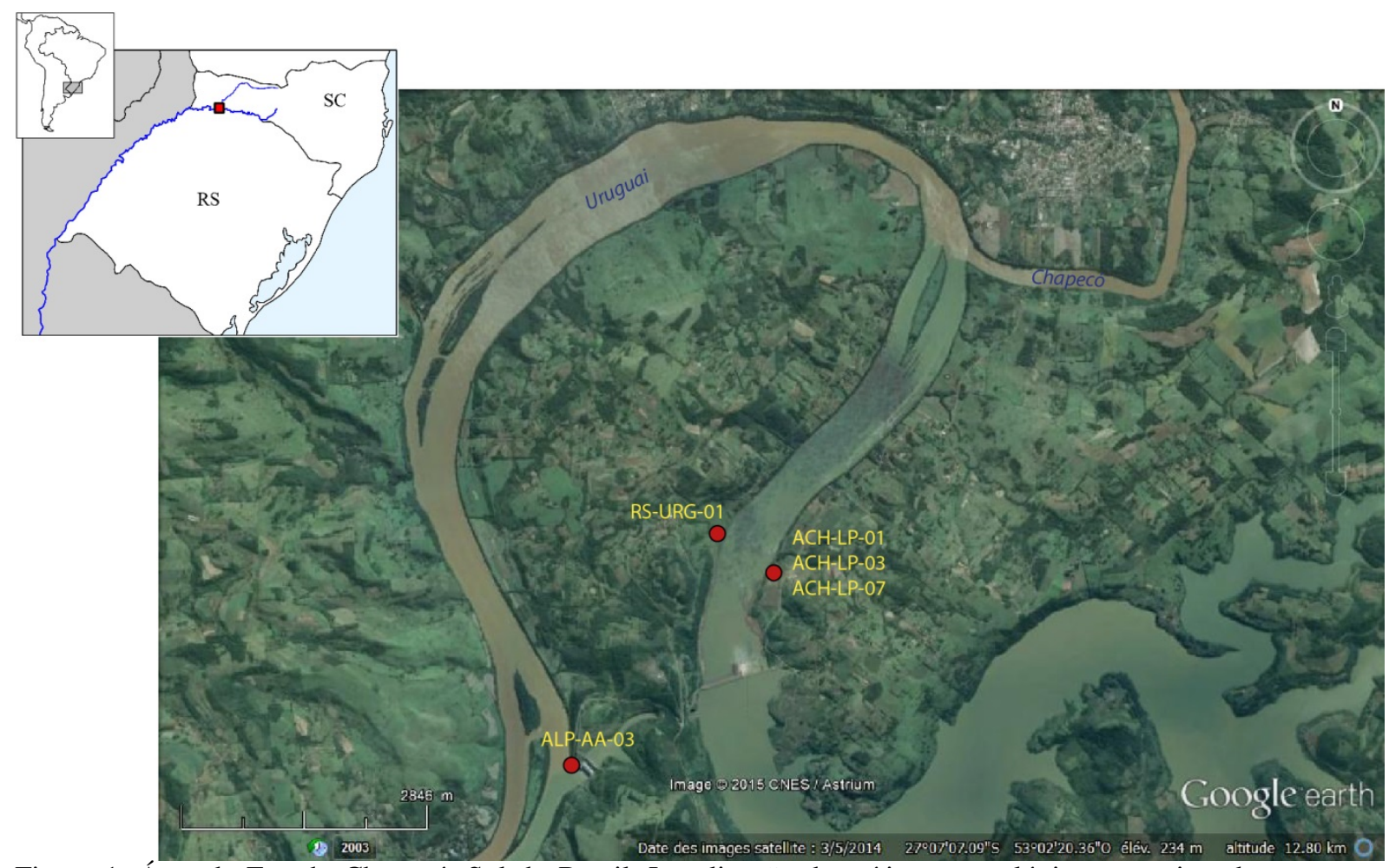

Figura 1. Área da Foz do Chapecó, Sul do Brasil. Localização dos sítios arqueológicos mencionados no texto (foto satélite: GoogleEarth).

Figure 1. Foz do Chapecó area, South Brazil. Location of archaeological sites mentioned in the text (satellite image: GoogleEarth).

Apesar da consistência destas informações, o entendimento completo dos esquemas operatórios ficava, até este momento, limitado pela ausência dos núcleos. A retomada das pesquisas de campo a partir de 2013, no âmbito de um projeto de pesquisa internacional sobre os povoamentos pré-históricos do alto e médio rio Uruguai, tem permitido preencher parte desta lacuna. Vários núcleos que correspondem a esta produção laminar foram encontrados em superfície, em áreas de erosão nas duas margens do rio Uruguai, nos sítios Linha Policial 7 (ACH-LP-07) e Uruguai 1 (RS-URG-01) (Figuras 1 e 2). O presente artigo tem como objetivo complementar o conhecimento sobre a concepção da debitagem laminar na região da Foz do Chapecó baseando-se nas propriedades da estrutura volumétrica desses núcleos. Para isso, apresentamos a seguir a análise tecnológica dos cinco núcleos encontrados nas coletas de superfície de 2013 e 2014.

\section{Contexto}

Devido ao importante potencial arqueológico da região da foz do Chapecó e à presença excepcional da debitagem laminar, pesquisas acadêmicas seguiram os trabalhos de salvamento a partir de 2013, nas áreas não destruídas pela construção da usina hidrelétrica Foz do Chapecó no rio Uruguai. Os sítios ALP-AA-03, ACH-LP-01 e ACH-LP-03 não existem mais. No entanto, próximo à área dos dois últimos foi encontrada grande quantidade 
de material lítico lascado e de cerâmica ${ }^{1}$, na margem direita do rio Uruguai. Trata-se de vestígios expostos recentemente, em consequência da forte erosão das duas margens, devido à modificação do regime das águas logo abaixo da barragem (Figura 2). As beiras recuam aos poucos pela ação do rio e os níveis arqueológicos vão sendo afetados. Assim, o material na superfície encontra-se relativamente próximo a sua posição original.

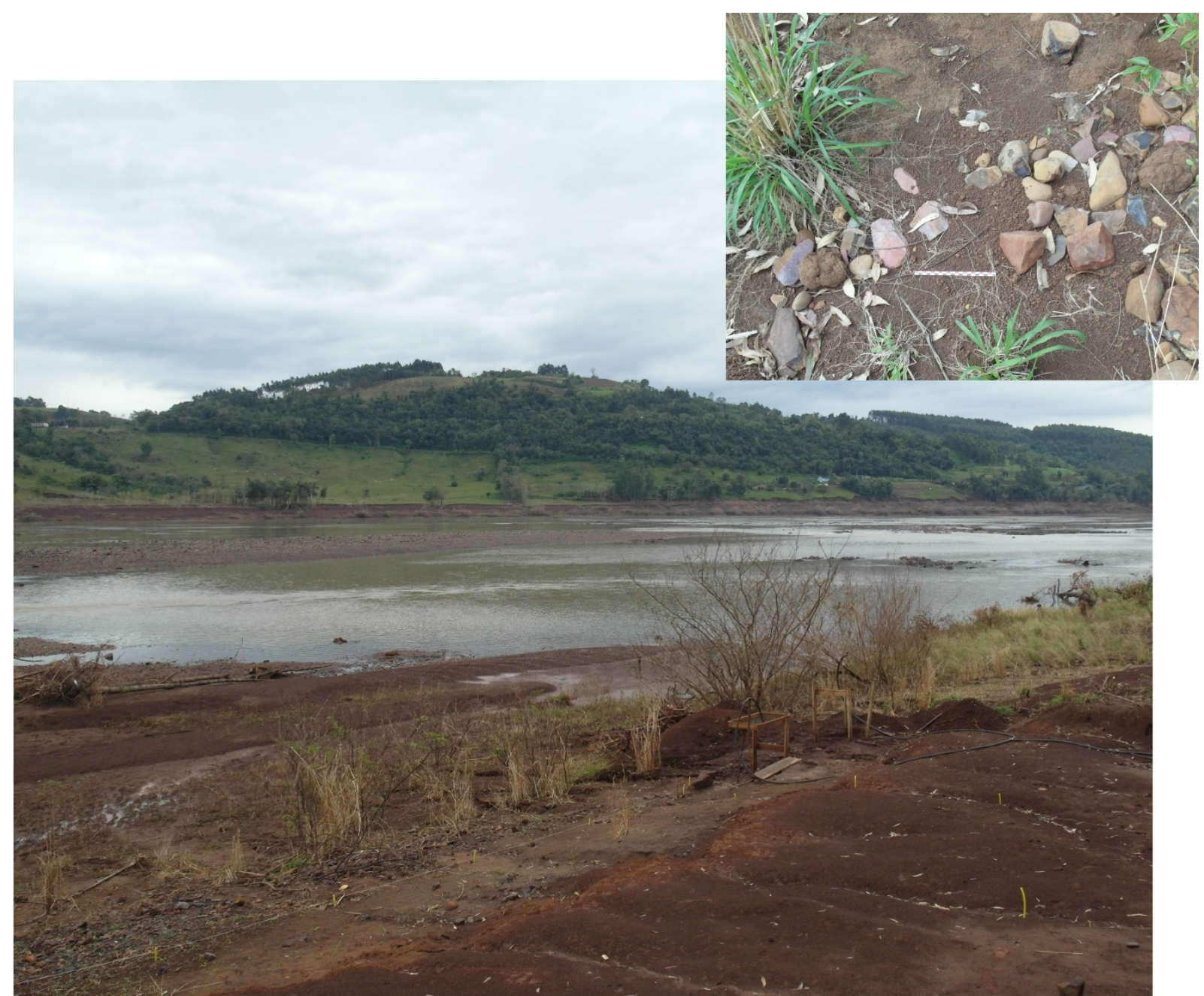

Figura 2. Vista das duas margens do rio Uruguai desde o patamar Linha Policial. No primeiro plano, vê-se ACHLP-07. O sítio RS-URG-01 encontra-se na margem oposta do rio. Em detalhe: superfície de erosão com artefatos no sítio ACH-LP-07.

Figure 2. View of the two sides of the Uruguay River from the Linha Policial platform. In the foreground, one can see ACH-LP-07. The site RS-URG-01 is in the other site of the river. In detail: erosion surface with artefacts in ACH-LP-07.

Esses vestígios em superfície permitiram definir o sítio Linha Policial 7 (ACH-LP-07), em Águas de Chapecó (Estado de Santa Catarina), que está sendo escavado desde 2014 (Figura 1). Tal intervenção evidenciou uma sequência arqueológica da transição PleistocenoHoloceno ao Holoceno recente. O período relativo à produção de lâminas, que nos interessa aqui, foi datado entre 10.500 e 9.500 cal. AP (Lourdeau et al. 2016). Corresponde a ocupações sucessivas de grupos de caçadores-coletores na beira do rio. As condições sedimentares no sítio não permitiram a preservação dos vestígios orgânicos. Assim, os modos de subsistência desses grupos não podem ser determinados diretamente. No entanto, a presença de várias pontas de projétil bifaciais nas áreas escavadas atesta de atividades de caça. 
Como nas pesquisas anteriores na região, a debitagem laminar é atestada em contexto somente pelos produtos, não por núcleos, e ela encontra-se associada a uma variedade impressionante de outros esquemas operatórios de façonnage e de debitagem (Lourdeau et al. 2016). Nas coletas de superfície das campanhas de 2013 e 2014, cerca de 1.000 vestígios arqueológicos de vários períodos foram encontrados. Entre eles, quatro núcleos laminares, descritos a seguir, foram reconhecidos (núcleos 1 a 4). Dado os resultados das escavações e as datações de ACH-LP-07, esses núcleos podem ser associados ao Holoceno inicial.

Do outro lado do rio Uruguai, na margem esquerda, um outro sítio arqueológico foi encontrado, Uruguai 1 (RS-URG-01), em Alpestre (Estado do Rio Grande do Sul) (Figura 1). Ele está sendo escavado desde 2015 e apresenta uma sequência arqueológica comparável com a de ACH-LP-07, ainda que mais rica que este último nos níveis relativos à segunda metade do Holoceno. Como do outro lado do rio, a base da sequência apresenta um conjunto arqueológico do Holoceno inicial com uma indústria lítica variada, inclusive lâminas. Na primeira coleta feita em 2014, foi encontrado um fragmento de núcleo laminar, apresentado a seguir (núcleo 5).

\section{Descrição dos núcleos}

Para a análise desses núcleos, baseamo-nos nos princípios metodológicos da abordagem tecnológica das indústrias líticas (Inizan et al. 1999). Enquanto abordagem conceitual, ela permite identificar os modos de produção, revelando os esquemas mentais que regem as atividades técnicas (Boëda 1991; Pelegrin 1995). Tais esquemas operatórios concretizam-se em cadeias operatórias, inseridas em um tempo e um espaço determinados (Karlin et al. 1991; Leroi-Gourhan 1964; Pelegrin et al. 1988). Nosso objetivo é identificar a estrutura dos núcleos laminares estudados e seu(s) método(s) de exploração. A análise procurou, em um primeiro tempo, entender a cronologia e a organização das retiradas sucessivas produzidas na superfície dos blocos. Isso foi possível a partir da leitura técnica dos estigmas de lascamento identificáveis nos núcleos (Inizan et al. 1999; Pelegrin 1995). A etapa de análise sucessiva à leitura técnica foi a hierarquização das informações. Nessa fase interpretativa, procuramos a intenção à origem de cada retirada efetuada e sua função dentro da cadeia operatória de produção das lâminas. De acordo com a localização e a organização das retiradas no volume e sua posição cronológica, identificamos vários grupos de retiradas, ou seja, várias "sequências", sendo elas relacionadas seja a fases de inicialização (a preparação do núcleo antes de sua exploração stricto sensu) ou a fases de produção dos objetivos da debitagem, isto é, as lâminas.

Cada núcleo é representado a seguir por um desenho convencional e um esquema diacrítico, os quais evidenciam as principais informações volumétricas e tecnológicas desses objetos (Dauvois 1976). Por convenção a disposição dos núcleos corresponde à orientação da série de lâminas, o plano de percussão sendo colocado para cima. Chamamos de "parte proximal” do núcleo a zona que contém o plano de percussão, e de "parte distal” a zona oposta.

Núcleo 1 (Figura 3): Foi feito a partir de um seixo de sílex de tamanho médio. O núcleo apresenta as seguintes dimensões: $11,4 \mathrm{~cm}$ de comprimento, $8,5 \mathrm{~cm}$ de largura e $6,5 \mathrm{~cm}$ de espessura. Pela superfície cortical ainda presente na parte distal do núcleo, nota-se que o seixo original não foi muito reduzido. A primeira operação foi de produzir o dorso por uma grande retirada oblíqua em relação ao eixo do seixo, e cujo negativo deixou uma grande superfície plana. Essa retirada foi seguida pela abertura do plano de percussão no polo adjacente ao negativo do dorso. A superfície de debitagem não apresenta nenhum negativo correspondente a uma fase de inicialização. Não significa que a mesma não aconteceu, mas pode ter sido apagada pelos negativos de lâminas que cobrem a totalidade dessa superfície. Um total de 8 
negativos de lâminas encontram-se visíveis no núcleo. Todas essas lâminas foram produzidas segundo o método unidirecional, a partir do plano de percussão obtido na fase de inicialização. Pelos estigmas de lascamento, especificamente os contra-bulbos bem marcados, pode-se identificar a técnica de lascamento destas lâminas como percussão direta com percutor de pedra (percussão interna). A parte distal do núcleo fraturou-se no decorrer da sequência de produção. A produção das lâminas aconteceu principalmente em uma superfície não muito convexa, estendendo-se nos dois lados do núcleo. Pelo esquema diacrítico, o ritmo da debitagem das lâminas organiza-se nos dois lados em direção ao centro da superfície de debitagem. A partir dos negativos visíveis, as lâminas produzidas têm entre 6 e $10 \mathrm{~cm}$ de comprimento.

Núcleo 2 (Figura 4): Trata-se de um núcleo reutilizado. O suporte inicial selecionado foi um bloco anguloso de arenito que já apresentava algumas retiradas, as quais são visíveis hoje como negativos patinados. A presença da pátina permite estabelecer que o núcleo primário foi abandonado certo tempo antes de ser reutilizado como núcleo laminar. Apresenta um comprimento de 13,5 cm, uma largura de $10 \mathrm{~cm}$ e uma espessura de $6,6 \mathrm{~cm}$. A fase de inicialização se limitou a produzir o plano de percussão em uma extremidade do bloco. Exceto três negativos patinados na parte distal, toda a superfície de debitagem é coberta por negativos de lâminas (bem sucedidas ou não), de modo que não se sabe se essa superfície passou por uma fase de preparação preliminar. O dorso do núcleo é naturalmente plano, formado por uma grande superfície de fratura. Após a inicialização, uma primeira sequência de lâminas foi efetuada segundo o método unidirecional, a partir do plano de percussão produzido. Pode-se efetuar uma subdivisão entre os produtos oriundos desta sequência: lâminas obtidas por percussão direta com percutor orgânico (percussão marginal) e lâminas obtidas com percussão interna com pedra. As primeiras foram destacadas no centro da superfície ( $\mathrm{n}^{\circ} 4,5$ e 1 no esquema diacrítico) enquanto as segundas foram feitas nos dois lados da superfície de debitagem (números 2 e 3 dos dois lados), com um alto grau de acidentes, pois nota-se um negativo refletido de um lado e um siret do outro. Segundo seus negativos, as três lâminas obtidas por percussão marginal orgânica têm entre 8 e $9 \mathrm{~cm}$ de comprimento. O ritmo de debitagem segue, no geral, o sentido anti-horário. Esse núcleo apresenta a especificidade da sua exploração ter sido finalizada pela debitagem de uma lâmina segundo uma direção oposta por percussão interna com pedra, a partir de um plano de percussão distal e à custa do dorso do núcleo, não da superfície de debitagem.

Núcleo 3 (Figura 5): Esse núcleo foi produzido a partir de um seixo de sílex. Ele tem 8,5 cm de comprimento, 6,8 $\mathrm{cm}$ de largura e 3,9 $\mathrm{cm}$ de espessura. O negativo II da fase de inicialização pode ser interpretado de duas formas diferentes: se trata ou de um negativo de grande retirada, ou de uma superfície de split, o seixo tendo sido aberto por percussão bipolar sobre bigorna. Os estigmas legíveis não permitem decidir entre essas duas possibilidades. Se for a primeira, o suporte inicial do núcleo é um seixo; se for a segunda, o suporte inicial é um fragmento de seixo. A primeira operação efetuada foi a abertura do plano de percussão em um dos polos do seixo, antes de produzir a grande superfície plana deixada pelo negativo (ou pela superfície de split) II. Essa superfície, junto com o negativo III, formam o dorso plano do núcleo. Depois da produção do dorso, a superfície de debitagem foi inicializada por pequenas retiradas centrípetas. As mesmas estão visíveis no lado esquerdo do núcleo. Essa inicialização pode ter sido utilizada também no lado direito, mas, se foi o caso, os negativos de produção de lâminas apagaram os negativos resultantes desta preparação. Em seguida, uma série de lâminas, de entre 6 e $8 \mathrm{~cm}$ de comprimento, foi produzida por percussão interna com pedra, segundo um método unidirecional. O ritmo da debitagem é alternante, de tipo 1-2-1-2. No final da sequência, a superfície de debitagem não apresentava mais uma convexidade longitudinal conveniente para a produção de lâminas, de modo que as duas últimas retiradas refletiram. 

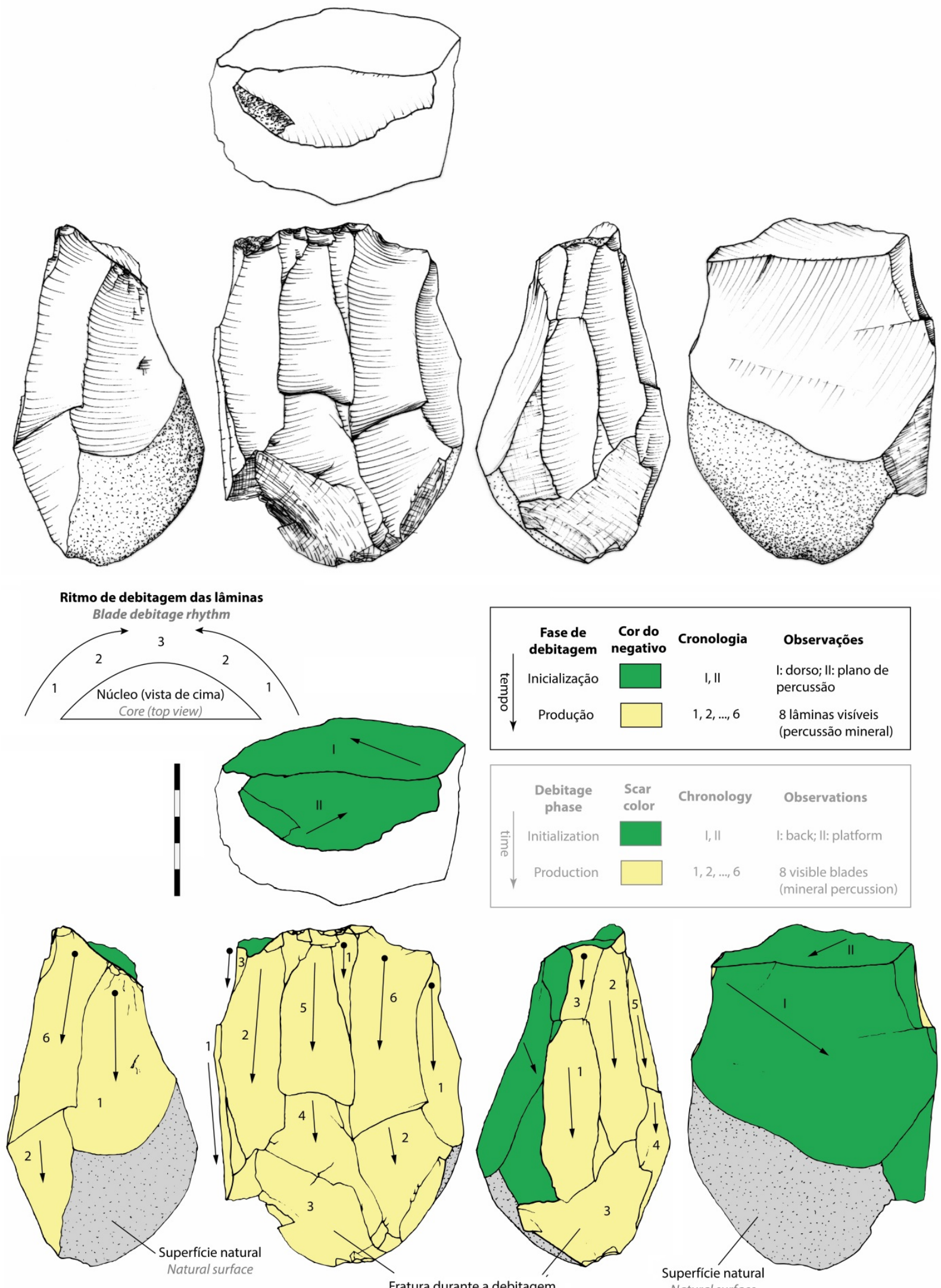

Fratura durante a debitagem
Fracture during debitage

Figura 3. Desenho e esquema diacrítico do núcleo 1, encontrado em ACH-LP-07.

Figure 3. Drawing and diacritic scheme of core 1, from ACH-LP-07. 

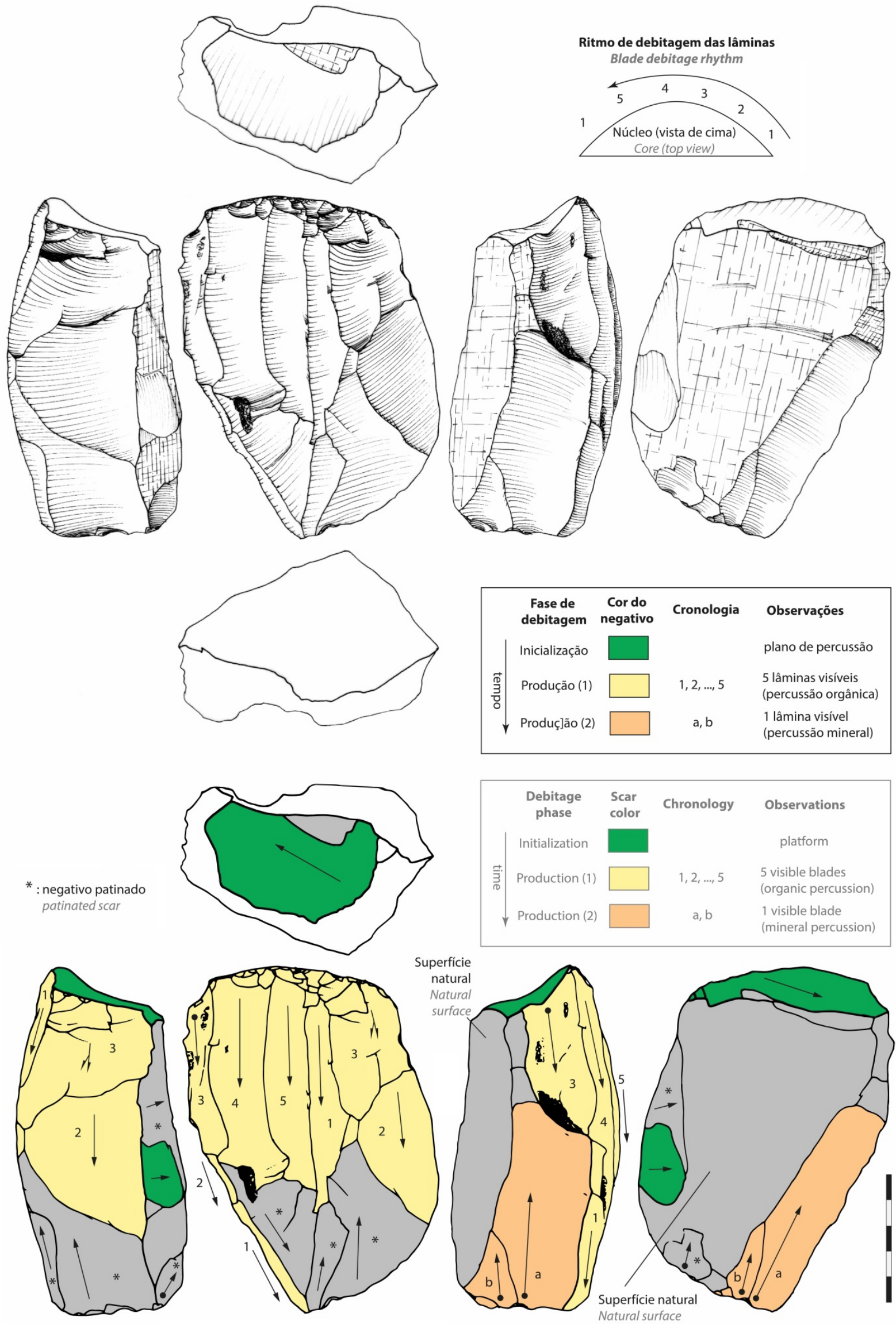

Figura 4. Desenho e esquema diacrítico do núcleo 2, encontrado em ACH-LP-07.

Figure 4. Drawing and diacritic scheme of core 2, from ACH-LP-07. 


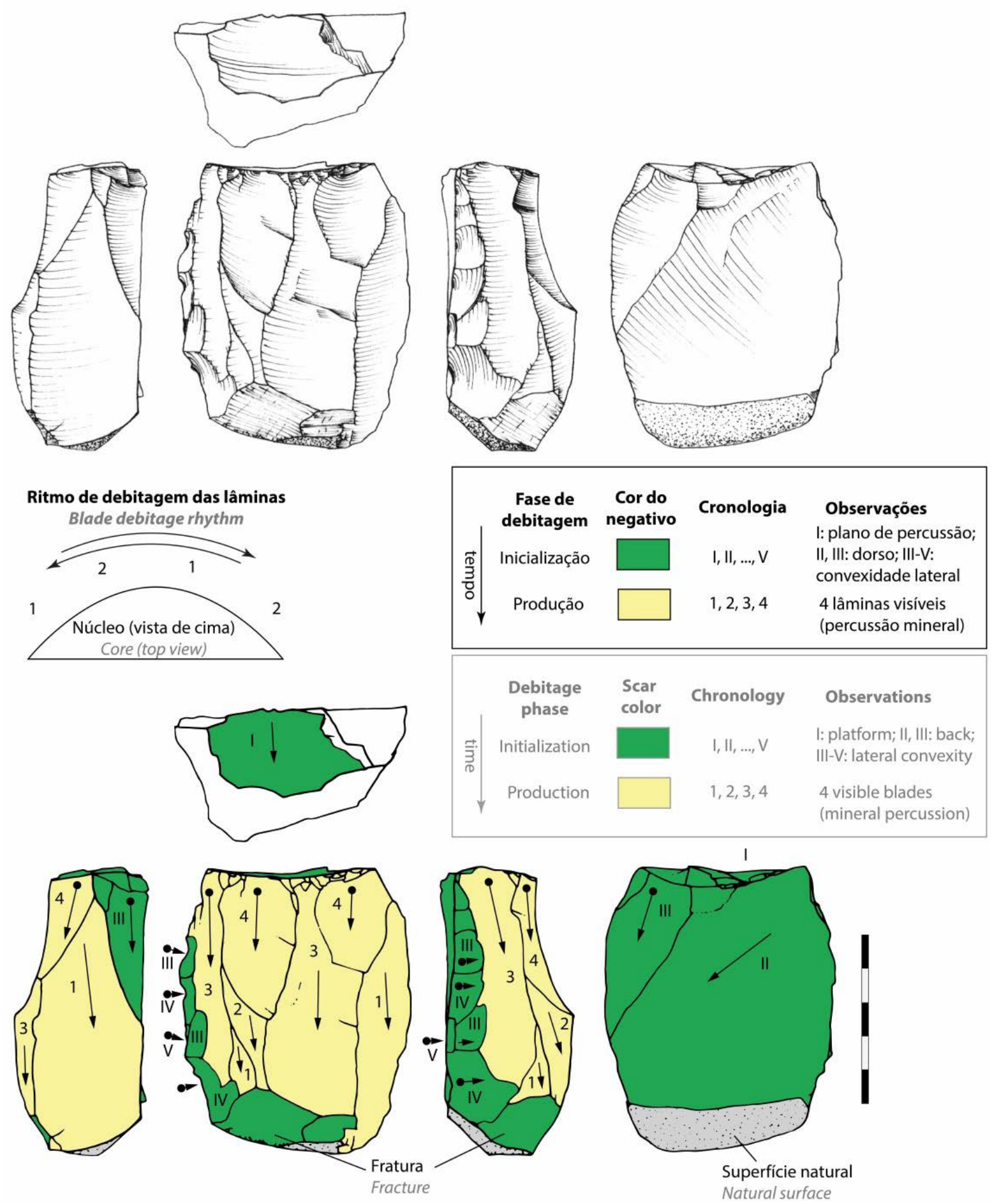

Figura 5. Desenho e esquema diacrítico do núcleo 3, encontrado em ACH-LP-07.

Figure 5. Drawing and diacritic scheme of core 3, from ACH-LP-07.

Núcleo 4 (Figura 6a): O suporte original desse núcleo de sílex foi muito modificado e não restou nenhuma parte natural na sua superfície. Assim, a morfologia do bloco original fica indeterminada. Seu comprimento é de $7,2 \mathrm{~cm}$, sua largura de $4,4 \mathrm{~cm}$ e sua espessura de $2,8 \mathrm{~cm}$. A fase de inicialização instalou o dorso plano por uma sequência de retiradas efetuada segundo uma direção transversal ao eixo do núcleo. Em seguida, o plano de percussão foi produzido por uma única retirada em um plano perpendicular ao do dorso, em uma extremidade do núcleo. O método de inicialização da superfície de debitagem permanece 
indeterminado, devido à retirada sucessiva das lâminas, que levaram os negativos anteriores. Os negativos das lâminas produzidas abrangem toda a superfície de debitagem e os lados do núcleo. Foram obtidas com um método unidirecional, segundo um ritmo anti-horário. Os negativos das lâminas são regulares, com estigmas sugerindo uma percussão interna com pedra. As últimas lâminas debitadas mediam entre 6 e $7 \mathrm{~cm}$ de comprimento.

Núcleo 5 (Figura 6b): Trata-se de um fragmento de núcleo cuja parte distal está faltando. O suporte original era um seixo de sílex. Seu comprimento, fraturado, é de $4 \mathrm{~cm}$, sua largura de 4,1 cm e sua espessura de 2,6 cm. Uma superfície cortical plana constitui o dorso do núcleo. O único negativo de preparação ainda presente é aquele que produziu o plano de percussão, na extremidade do núcleo. Na superfície de debitagem, negativos de lâminas produzidas por percussão interna com pedra, segundo um método unidirecional. O ritmo de produção organiza-se, de forma geral, no sentido anti-horário.

\section{Síntese: a concepção da debitagem laminar na região da Foz do Chapecó}

\subsection{Os aportes dos núcleos}

A análise tecnológica dos cinco núcleos permite determinar a estrutura volumétrica que rege essa produção laminar. Efetivamente, apesar de uma variabilidade evidente, a debitagem de lâminas na região da Foz do rio Chapecó é a manifestação de um único conceito, que se manifesta na estrutura dos núcleos pelas seguintes características:

- O núcleo organiza-se em duas superfícies distintas: um dorso plano e uma superfície de debitagem convexa, mas relativamente achatada, de modo que o núcleo apresenta uma secção transversal em forma de “D”. Em uma extremidade encontra-se uma pequena superfície que corresponde ao plano de percussão para a produção das lâminas.

- A fase de produção é sempre precedida por uma fase de inicialização do núcleo. O plano de percussão é instalado durante esta etapa. É produzido por uma única grande retirada. Em função das propriedades naturais do volume inicial, o dorso é obtido seja no momento da seleção do suporte do núcleo (núcleos 2 e 5), seja por uma preparação parcial (núcleo 1) ou completa (núcleos 3 e 4). Quando foi obtida na fase de inicialização, a superfície plana do dorso resulta de um ou vários grandes negativos de retiradas produzidas segundo uma direção perpendicular ao eixo longitudinal do núcleo. Os estigmas da fase de inicialização da superfície de debitagem são geralmente retirados pela fase de produção das lâminas, de forma que não restam nos núcleos informações relativas a essa preparação. Entre os núcleos analisados, apenas no núcleo 3 subsistem negativos de inicialização, no lado esquerdo. São negativos centrípetos produzidos a partir do dorso do núcleo, para instalar a convexidade lateral. A estrutura do núcleo, com um dorso plano, favorece esse tipo de inicialização ou reinicialização parcial ou completa por retiradas centrípetas.

- As lâminas são sempre produzidas segundo um método unidirecional paralelo, a partir do plano de percussão obtido na fase de inicialização. O ritmo da debitagem, ou seja, a organização da sequência de debitagem das lâminas, pode variar bastante, mas nota-se certa predominância do ritmo seguindo o sentido anti-horário (núcleos 2, 4 e 5).

A variabilidade da produção refere-se principalmente às técnicas de lascamento. Pelos estigmas legíveis na superfície dos blocos, a inicialização do núcleo é sempre feita por percussão interna com pedra, mas a produção das lâminas pode ser por percussão interna com pedra (núcleos 1, 3, 4 e 5) ou por percussão marginal orgânica (núcleo 2). Essa variabilidade de técnica está ligada à medida do ângulo formado entre o plano de percussão e a superfície de debitagem. O mesmo encontra-se bastante aberto, quase reto, nos núcleos com percussão interna por pedra (núcleos 3, 4 e 5) e relativamente fechado quando se trata de percussão marginal orgânica (núcleo 2). 

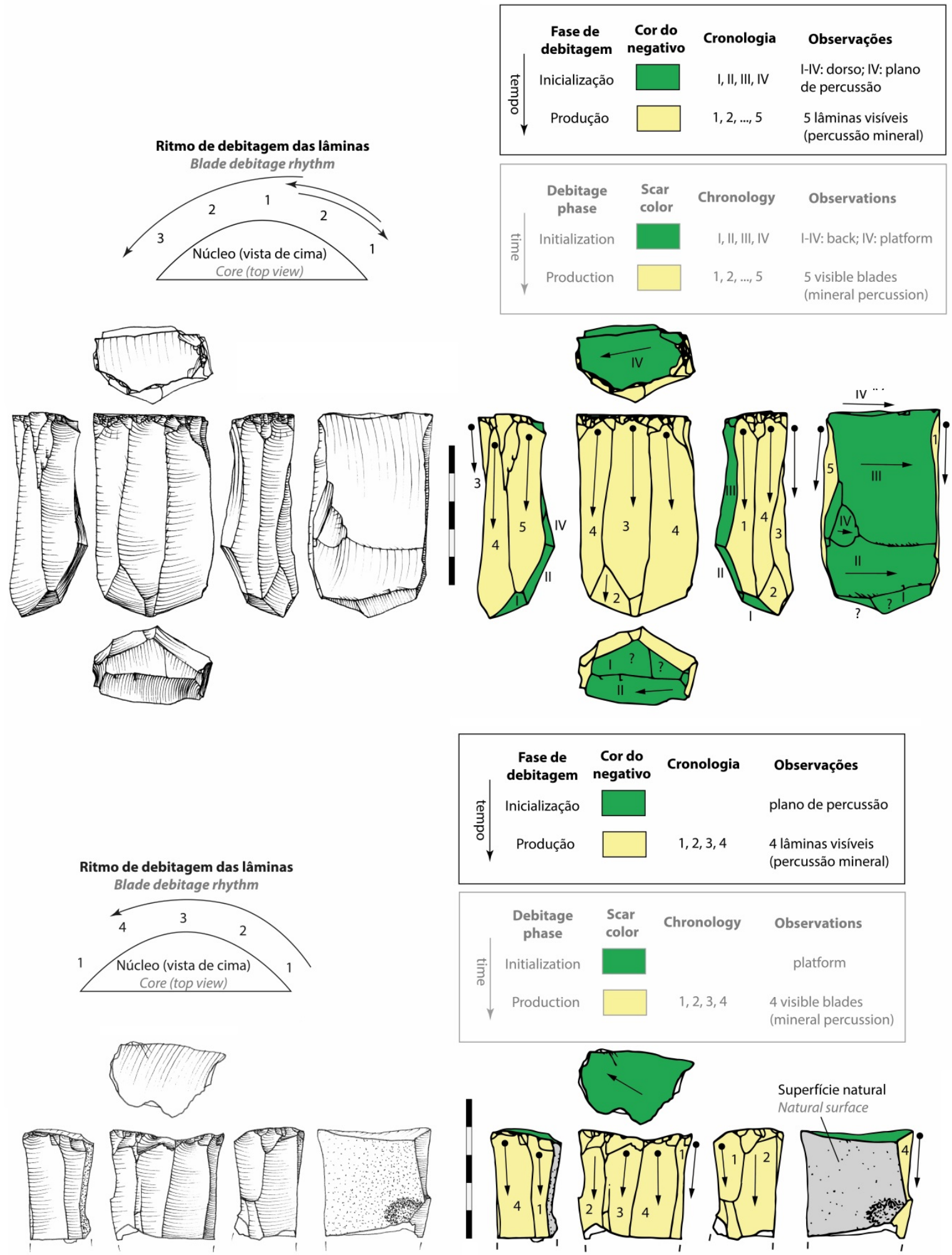

Figura 6. a. Desenho e esquema diacrítico do núcleo 4, encontrado em ACH-LP-07; b. Desenho e esquema diacrítico do núcleo 5, encontrado em RS-URG-01.

Figure 6. a. Drawing and diacritic scheme of core 4, from ACH-LP-07; b. Drawing and diacritic scheme of core 5, from RS-URG-01

Além disso, a variabilidade das dimensões dos núcleos (de 13,5 a 7,2 cm de comprimento) tem necessariamente consequências no comprimento das lâminas obtidas (entre 6 e $10 \mathrm{~cm}$, segundo a medida dos negativos preservados). 


\subsection{Voltando às lâminas}

As informações técnicas fornecidas por esses núcleos dos sítios ACH-LP-07 e RSURG-01 são complementares mas em total concordância com aquelas obtidas na análise das lâminas oriundas das escavações de ACH-LP-01, ACH-LP-03 e ALP-AA-03 (Lourdeau et al. 2014; Hoeltz et al. 2015). A existência de um método de produção unidirecional das lâminas, de uma inicialização centrípeta da superfície de debitagem (que implica um núcleo organizado em duas superfícies distintas), a ocorrência da percussão interna com pedra, bem como a percussão marginal orgânica, são elementos que tinham sido evidenciados pela análise diacrítica das lâminas. Isso aponta uma compatibilidade técnica completa entre as lâminas produzidas e os núcleos encontrados na área da Foz do Chapecó. As estrutura volumétrica hipotética proposta depois da análise das lâminas (Lourdeau et al. 2014; Hoeltz et al. 2015) corresponde perfeitamente àquela dos núcleos descobertos posteriormente. A análise dos núcleos complementa nosso conhecimento especificamente no que diz respeito ao dorso, cujas características não podiam ser determinadas a partir das lâminas. Por outro lado, a análise das lâminas possibilita mais informação quanto aos métodos de inicialização e reinicialização, já que na superfície dos núcleos só ficaram registrados os últimos momentos da debitagem. Conforme os resultados das análises publicadas, os negativos das faces superiores das lâminas demonstram o uso de um método de inicialização centrípeto, em complemento com as propriedades já presentes naturalmente na superfície de debitagem. De forma pontual, e somente para as reinicializações, um método centrífugo, baseado na instalação de cristas, pode ter sido usado.

Com esses dois estudos complementares das lâminas e dos núcleos laminares, atingimos uma compreensão relativamente completa do sistema de produção desses suportes na região da foz do Chapecó durante o Holoceno inicial, única ocorrência de debitagem laminar bem documentada até o momento no Brasil. Os próximos passos das pesquisas consistirão em entender essa produção específica dentro do sistema técnico geral no qual se insere, pela análise dos outros esquemas de produção associados e por uma abordagem tecnofuncional do instrumental confeccionado. As pesquisas em andamento em ACH-LP-07 e RS-URG-01 permitirão uma percepção diacrônica destas produções. A uma escala regional, agora é importante compreender as implicações desses novos dados técnicos no âmbito da préhistória do Sul do Brasil, que tende, em geral, a ser uniformizada pela noção de "tradição Umbu”. Por fim, uma abordagem comparativa ampla poderá, no futuro, fornecer orientações para entender o desenvolvimento e os vários aspectos do fenômeno laminar no cone sul da América meridional durante a primeira metade do Holoceno.

\section{Agradecimentos}

Essa pesquisa integra-se no projeto "Povoamentos pré-históricos do alto rio Uruguai”, que recebe apoio financeiro do Ministère des Affaires Etrangères et du Développement International da França. É fruto de uma parceria entre as seguintes instituições: o Centro de Memória do Oeste de Santa Catarina-Unochapecó, o Muséum National d’Histoire Naturelle de Paris e o Setor de Arqueologia/Laboratório de Arqueologia Pedro Ignácio Schmitz da Universidade do Extremo Sul Catarinense. Agradecemos a Scientia Consultoria Científica, o Instituto Goiô-En e o Consórcio Foz do Chapecó pelo apoio a essa pesquisa. Somos gratos a João Carlos Moreno de Sousa e Astolfo Gomes de Mello Araujo pela organização desse número especial em língua portuguesa. As observações feitas por dois pareceristas anônimos contribuíram a melhorar o texto original do artigo. 


\section{Referencias}

Barros, P., Martínez, G., \& Gutiérrez, M. 2014, Análisis de los materiales líticos del sitio Paso Otero 4 (Partido de Necochea, provincia de Buenos Aires). Avances en el conocimiento de las estrategias tecnológicas en el curso medio del río Quequén Grande para el Pleistoceno tardío-Holoceno. Relaciones de la Sociedad Argentina de Antropología, 39(1): 119-144. (em espanhol; in Spanish) (“Análises dos materiais líticos de Paso Otero 4 (Partido de Necochea, Província de Buenos Aires). Avanços no conhecimento de estratégia tecnológicas no curso médio do rio Quequén Grande para o Pleistoceno final-Holoceno"; "Analysis of the lithic assemblages from Paso Otero 4 (Necochea Disctrict, Buenos Aires Province). Advances in the knowledge of technological strategies in the middle basin of the Quequén Grande River during Late PleistoceneHolocene”) URL: http://www.saantropologia.com.ar/textos/analisis-de-los-materialesliticos-del-sitio-paso-otero-4-partido-de-necochea-provincia-de-buenos-aires

Boëda, E. 1991, Approche de la variabilité des systèmes de production lithique des industries du Paléolithique inférieur et moyen : Chronique d'une variabilité attendue. Techniques et culture, 17, 18: 37-79. (em francês; in French) (“Abordagem da variabilidade dos sistemas de produção lítica das indústrias do Paleolítico inferior e médio: Crônica de uma variabilidade esperada"; "Approach to the variability of the lithic production systems of the Lower and Middle Paleolithic industries: Chronicle of an expected variability”) doi:10.4000/tc.685

Caldarelli, S. (Ed.), 2010, Arqueologia preventiva na UHE Foz do Chapecó, SC/RS. Relatório Final. Unpublished report, Scientia Consultoria Científica, Florianópolis, 875 p. (in Portuguese) ("Preventative Archaeology in Foz do Chapecó hydroelectric plant (Santa Catarina/Rio Grande do Sul). Final report”)

Dauvois, M. 1976, Précis de dessin dynamique et structural des industries lithiques préhistoriques, Pierre Fanlac, Périgueux, 263 p. (em francês; in French) ("Compêndio de desenho dinâmico e estrutural das indústrias líticas pré-históricas”; "Résumé of dynamic and structural drawing of prehistoric lithic industries”)

Hermo, D., Magnin, L. 2012, Blade and bifacial technology in Mid-Holocene occupations at Deseado Massif, Santa Cruz province, Argentina. Quaternary International, 256: 71-77. (em inglês) ("Tecnologia laminar e bifacial em ocupações do Holoceno médio no Maciço Deseado, Província de Santa Cruz, Argentina”) doi:10.1016/j.quaint.2011.09.034

Hoeltz, S. E., \& Brüggemann, A. A. 2011, As indústrias líticas na área da UHE Foz do Chapecó, oeste catarinense: Antiguidade, estratégia tecnológica e variabilidade. In: Antes do Oeste Catarinense: Arqueologia dos povos indígenas (Carbonera, M., Schmitz, P. I., Eds.). Editora Argos, Chapecó: p.105-136. (in Portuguese) (“Lithic industries in the Foz do Chapecó hydroelectric plant area, West Santa Catarina: Antiquity, technological strategy and variability")

Hoeltz, S. E. \& Brüggemann, A. A., 2014, As indústrias líticas na bacia do Prata, oeste catarinense: Antiguidade, estratégia tecnológica e variabilidade. In: Peuplement de l'Amérique du Sud: l'apport de la technologie lithique, (Farias, M., Lourdeau, A., Eds.). Archéoéditions, Prigonrieux: p.123-145. (in Portuguese) ("Lithic industries in the Prata River basin: Antiquity, technological strategy and variability”) 
Hoeltz, S. E., Lourdeau, A. \& Viana, S. 2015, Um novo conceito de lascamento no sul do Brasil: Debitagem laminar na foz do rio Chapecó (SC/RS). Revista do Museu de Arqueologia e Etnologia, 25: 3-19. (in Portuguese) (“A new flaking concept in South Brazil: Blade débitage in the mouth of Chapecó river (Santa Catarina/Rio Grande do Sul)”) doi:10.11606/issn.2448-1750.revmae.2015.114852

Hoguin, R., 2013, Evolution et changements techniques dans les sociétés de chasseurscueilleurs de la Puna Sèche des Andes Centrales-Sud: Technologie lithique dans la localité de Susques durant l'Holocène ancien et moyen. $\mathrm{PhD}$ thesis, Universidad de Buenos Aires and Université Paris Ouest Nanterre, Buenos Aires and Nanterre, 278 p. (em francês; in French) ("Evolução e mudanças técnicas em sociedades de caçadorescoletores da Puna Seca dos Andes Centrais e Sul: Tecnologia lítica em Susques durante o Holoceno inicial e médio"; "Evolution and technical changes in the hunter-gatherers societies of the Dry Puna of South-Central Andes : Lithic technology in the area of Susques during the early and middle Holocene") URL: http://www.theses.fr/2013PA100102

Inizan, M.-L., Reduron, M., Roche, H. \& Tixier, J. 1999, Technology and terminology of knapped stone, Préhistoire de la pierre taillée Vol. 5. Cercle de recherche et d'études préhistoriques, Nanterre, 189 p. (em inglês) ("Tecnologia e terminologia da pedra lascada”)

Karlin, C., Bodu, P. \& Pelegrin, J. 1991, Processus techniques et chaînes opératoires. Comment les préhistoriens s'approprient un concept élaboré par les ethnologues. In: Observer l'action technique. Des chaînes opératoires, pour quoi faire? (Balfet, H., Ed.), CNRS (Centre national de la recherche scientifique) Éditions, Paris: p. 101-118. (em francês; in French) ("Processos técnicos e cadeias operatórias. Como os préhistoriadores se apropriaram de um conceito elaborado pelos etnólogos”; “Technical processes and chaînes opératoires. How prehistorians appropriate a concept elaborated by ethnologists")

Leroi-Gourhan, A. 1964, Le geste et la parole II : La mémoire et les rythmes. Albin Michel, Paris, 288 p. (em francês; in French) (“O gesto e a palavra II: Memória e ritmos”; “Gesture and speech II: Memory and rhythms”)

Lourdeau, A., Hoeltz, S. \& Viana, S. A. 2014, Early Holocene blade technology in southern Brazil. Journal of Anthropological Archaeology, 35: 190-201. (em inglês) ("Tecnologia laminar no Holoceno inicial no sul do Brasil”) doi:10.1016/j.jaa.2014.06.003

Lourdeau, A., Carbonera, M., Santos, M. C. P., Hoeltz, S., Fontugne, M., Hatté, C., Silva, S. F. S. M., Rosina, P., Lucas, L. O., Da Costa, C. Foucher, A., Ramalho, J. B., Kuczkovski, F., Campos, J. B., Viana, S. A. \& Herberts, A. 2016, Pré-história na foz do rio Chapecó. Cadernos do CEOM, 29(45): 220-242. (in Portuguese) ("Prehistory in the mouth of the Chapecó River”) doi:10.22562/2016.45.09

Nami, H. 1996, Nota sobre la presencia de “microhojas” y lascas de arista en los niveles medios de Piedra del Aguila 11. Praehistoria, 2: 101-112. (em espanhol; in Spanish) ("Nota sobre a presença de "microlâminas" e lascas de gume nos níveis médios de Piedra del Aguilla 11"; "Note on the presence of "microblades" and edge flakes in the middle levels of Piedra del Aguila 11”) 
Pelegrin, J. 1995, Technologie lithique : Le Châtelperronien de Roc-de-Combe (Lot) et de La Côte (Dordogne). Cahiers du Quaternaire Vol. 20. CNRS Éditions, Paris, 297 p. (em francês; in French) ("Tecnologia lítica : O Chatelperronense de Roc-de-Combe (lot) e de La Côte (Dordonha)"; "Lithic technology: The Châtelperronian from Roc-de-Combe (Lot) and La Côte (Dordogne)”)

Pelegrin, J., Karlin, C. \& Bodu, P. 1988, “ Chaînes opératoires ” : Un outil pour le préhistorien. Technologie préhistorique, Notes et Monographies Techniques, 25: 55-62. (em francês; in French) ("Cadeias operatórias: Uma ferramenta para o pré-historiador”; “Chaînes opératoires: A tool for the prehistorian”)

Suárez, R. 2011, Movilidad, acceso y uso de ágata translúcida por los cazadores-recolectores tempranos durante la transición Pleistoceno Holoceno en el Norte de Uruguay (ca. 11,000-8500 A.P). Latin American Antiquity, 22: 359-383. (em espanhol; in Spanish) ("Mobilidade, acesso e uso de ágata translúcida pelos caçadores-coletores antigos durante a transição Pleistoceno-Holoceno no norte do Uruguai”; "Mobility, access and use of translucent agate by early hunter-gatherers during the Pleistocene Holocene transition in North Uruguay (ca. 11,000-8500 BP)”) doi:10.7183/1045-6635.22.3.359 


\title{
Blade debitage in Southern Brazil: We have cores!
}

\author{
Antoine Lourdeau ${ }^{1}$, Mirian Carbonera ${ }^{2}$, Sirlei Hoeltz ${ }^{3}$, Marcos C. Pereira \\ Santos ${ }^{4}$, Lívia de Oliveira e Lucas ${ }^{5}$, Amélie Da Costa ${ }^{6}$, Sibeli Viana ${ }^{7}$
}

1. Muséum National d’Histoire Naturelle \& Universidade Federal de Sergipe. Musée de l’Homme, Département de Préhistoire, 17, place du Trocadéro, 75116 Paris, France. Email: antoine.lourdeau@mnhn.fr

2. Centro de Memória do Oeste de Santa Catarina (CEOM) \& Ciências Ambientais, Unochapecó, Rua John Kennedy, 279E, Chapecó - SC, Brazil. Email: mirianc@unochapeco.edu.br

3. Archaeo Pesquisas Arqueológicas, Av. Carandaí, 99, Parque Georgia, 78.085-485, Cuiabá - MT, Brazil. Email: sirlei@archaeo.com.br

4. Università Degli Studi di Ferrara \& Universidade do Extremo Sul Catarinense. Rodovia Governador Jorge Lacerda, km 4,5 - Bairro Sangão. 88805-350 - Caixa Postal 3167, Criciúma - SC, Brazil.

Email: marcoscesar@unesc.net

5. Universidade Federal de Sergipe \& Universidade Federal do Vale do São Francisco. UNIVASF, rua João Ferreira dos Santos, s/nº, Campestre, 64770-000, São Raimundo Nonato - PI, Brazil. Email: livia.lucas@univasf.edu.br

6. Université de Paris Ouest Nanterre La Défense. UMR 7041 ArScAn-ANTET. Maison de l’Archéologie et de

l’Ethnologie, 21 allée de l’Université, 92023 Nanterre, France. Email : amelie.da-costa@mae.u-paris10.fr

7. Pontifícia Universidade Católica de Goiás, Instituto Goiano de Pré-História e Antropologia, Praça

Universitária, n. 1440, Universitário, 74605-220, Goiania, GO - Brazil. Email: sibeli@pucgoias.edu.br

\begin{abstract}
:
Blade debitage is a characteristic production pattern of specific periods and regions during Prehistory. In South America, it is well documented in Argentina, in contexts dating back to early Holocene. In Brazil, it was unknown until 2006, when it was found in three archaeological sites (ACH-LP-01, ACH-LP-03, ALP-AA-03) in the Foz do Chapecó area, upper Uruguay River, South Brazil, in layers from early Holocene. It was associated there with a great variety of other production systems: bifacial shaping of projectile points and large tools, unipolar debitage of large flakes, bipolar debitage of tiny flakes. Silicified sandstone, chalcedony and hyaline quartz are the main used raw materials. To this day, only the products of this debitage, the blades, had been discovered. Cores were lacking, which prevented a complete understanding of the flaking process. Due to the resumption of research in the region from 2013, it was possible to find those cores during surveys in the two following archaeological sites: ACH-LP-07 and RS-URG-01, located on both sides of the Uruguay river, near the mouth of the Chapecó river. We describe in this article five of these cores (4 from ACH-LP-07 and 1 from RS-URG-01). Their analyses demonstrate that this blade debitage complies with only one concept. The core volumetric structure that is the result of this concept displays the following features: 1) Cores have two surfaces: a flat back and a convex but relatively flattened flaking surface, so that they have a general " $D$ " shaped transversal section. At one end, a small surface acts as striking platform during the production of the blades; 2) Debitage begins with a initialization phase of the core during which the striking platform is produced by a large removal. According to the natural properties of the initial volume, the back of the core is obtained either during the selection of the blank, and then it is left in his natural state, or by a preparation by one or more large transversal removals. When present, the scars of the initialization phase of the flaking surface indicate the use of a centripetal method. This preparation is made possible by the acute angle of the peripheral ridge formed by the intersection of the back and the flaking surface ; 3) Blades are always produced by a unidirectional parallel method. Production variability is mainly related to the flaking technique: both internal percussion by stone and marginal percussion by organic percussor were used to get the blades.
\end{abstract}


Technical information provided by the cores are complementary and in accordance with those from the previous analyses of the blades from the same area. With these two studies it was possible to reach a relatively exhaustive understanding of this production system in the upper Uruguay River during early Holocene, the only well documented blade debitage in Brazil known until now. Future research will explore the interactions between this production and the other debitage and shaping methods inside this early Holocene technological system. To do this, we will develop a comprehensive study of all lithic artefacts from the archaeological layers in which blade production was identified. In a wider scale, a comparative approach with Argentinian and Uruguayan prehistoric blade productions will allow to better understand the development of the blade phenomenon in the southern cone of South America.

Keywords: blade production; prehistory of Brazil; Uruguay River; Early Holocene; technological analysis; Foz do Chapecó 